\title{
Recent results on B-Physics and Quarkonia with the ATLAS detector
}

\section{Artem Maevskiy ${ }^{*}$}

On behalf of the ATLAS collaboration

E-mail: Artem.Maevskiy@cern.ch

The paper describes several measurements recently completed by ATLAS: (a) the decay of $\Lambda_{b}^{0}$ into $\Lambda^{0}$ and $\psi(2 S)$, observed for the first time and compared to the decay into $\Lambda^{0}$ and $J / \psi$; (b) the decay of $B_{c}^{+}$into $J / \psi D_{s}^{+}$and $J / \psi D_{s}^{*+}$, including polarisation measurement for the latter channel; (c) the measurement of the ratio of the $b$-quark fragmentation fractions $f_{s} / f_{d}$, which complements a previous measurement of LHCb over a different range of $p_{\mathrm{T}}$ and $y$; (d) $B^{+}$mass reconstruction in the decay of $B^{+}$to $J / \psi$ and $K^{+}$at ATLAS at $13 \mathrm{TeV} p p$ collisions; (e) measurement of the differential non-prompt $J / \psi$ production fraction at $13 \mathrm{TeV}$.

XXIV International Workshop on Deep-Inelastic Scattering and Related Subjects

11-15 April, 2016

DESY Hamburg, Germany

\footnotetext{
*Speaker.

${ }^{\dagger}$ This work was partially supported by RFBR, research project No. 15-02-08133.
} 


\section{Introduction}

Since 2015 the Large Hadron Collider (LHC) has started its Run-2 after a two year shutdown dedicated to upgrade its facilities. In the new run LHC operates at a new centre-of-mass energy of $p p$ collisions $\sqrt{s}=13 \mathrm{TeV}$, compared to $8 \mathrm{TeV}$ at the end of Run- 1 . The pile-up conditions, as well as the luminosity, have also increased. One of the major ATLAS [1] upgrades was the insertion of a new layer of pixel detectors between the existing detector and the beam pipe intended to improve the vertexing resolution.

An excellent ATLAS performance under the new operation conditions has been shown by the first physics results with the new data, two of which $-B^{+}$-meson mass measurement and non-prompt $J / \psi$ production fraction measurement - are presented in this paper. This paper also presents three recent results using Run-1 data: $\Gamma\left(\Lambda_{b}^{0} \rightarrow \psi(2 S) \Lambda^{0}\right) / \Gamma\left(\Lambda_{b}^{0} \rightarrow J / \psi \Lambda^{0}\right)$ branching ratio measurement, polarisation and branching ratio measurement for the $B_{c}^{+} \rightarrow J / \psi D_{s}^{(*)+}$ decays and the measurement of the ratio of the $b$-quark fragmentation fractions $f_{s} / f_{d}$.

\section{Measurement of the branching ratio $\Gamma\left(\Lambda_{b}^{0} \rightarrow \psi(2 S) \Lambda^{0}\right) / \Gamma\left(\Lambda_{b}^{0} \rightarrow J / \psi \Lambda^{0}\right)$}

The observation of the $\Lambda_{b}^{0} \rightarrow \psi(2 S) \Lambda^{0}$ decay and the branching ratio measurement of $\Gamma\left(\Lambda_{b}^{0} \rightarrow \psi(2 \mathrm{~S}) \Lambda^{0}\right) / \Gamma\left(\Lambda_{b}^{0} \rightarrow J / \psi \Lambda^{0}\right)$ was done with $20.6 \mathrm{fb}^{-1}$ of $p p$ collision data at $\sqrt{s}=8 \mathrm{TeV}$ [2]. Events with at least two oppositely charged muons were selected for this analysis. The two muon tracks were fitted into a common vertex to form a $J / \psi$ or $\psi(2 S)$ vertex. Two more tracks were also fitted with a proton and pion mass hypotheses to a separate vertex to form $\Lambda^{0}$. The four tracks were then simultaneously refitted with a requirement on the combined momentum of the two hadron tracks to point to the dimuon vertex. Fits with $B^{0} \rightarrow J / \psi K_{S}^{0}$ and $B^{0} \rightarrow \psi(2 S) K_{S}^{0}$ decay topologies were also applied to each track quadruplet successfully fitted with $\Lambda_{b}^{0}$ topology to control the $B^{0}$ reflections. Figure 1 shows the invariant mass distributions $m\left(\psi(2 S) \Lambda^{0}\right)$ and $m\left(\psi(2 S) K_{S}^{0}\right)$.

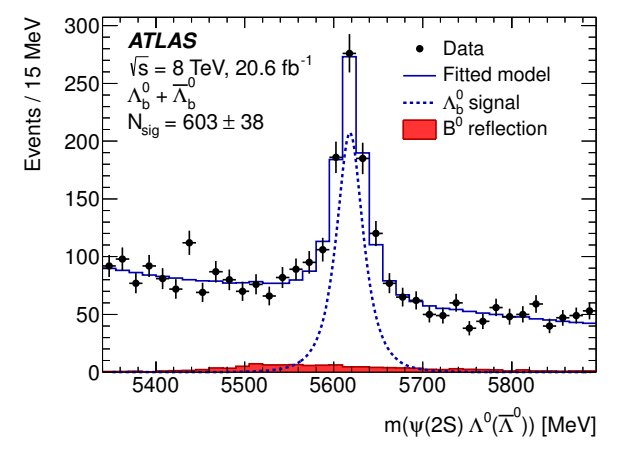

(a)

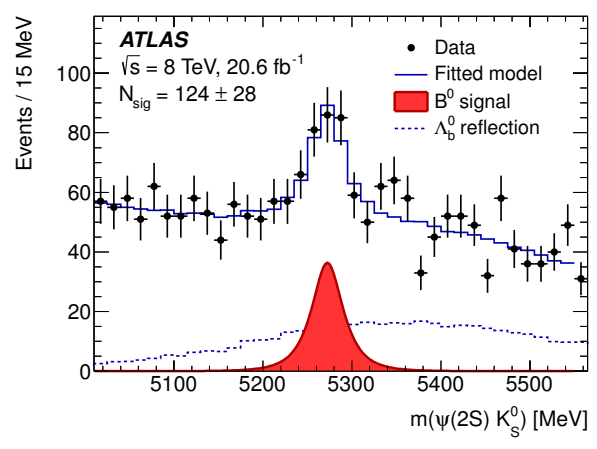

(b)

Figure 1: The invariant mass distributions for the combined sample of the selected $\Lambda^{0}$ and $\overline{\Lambda^{0}}$ candidates obtained after their fits to $\Lambda_{b}^{0} \rightarrow \psi(2 \mathrm{~S}) \Lambda^{0}$ (a) and $B^{0} \rightarrow \psi(2 \mathrm{~S}) K_{S}^{0}$ (b) topologies [2].

The $m\left(J / \psi \Lambda^{0}\right)$ and $m\left(J / \psi K_{S}^{0}\right)$ distributions are simultaneously fitted using analytical functions for the signal and non-resonant background components and MC templates for the mutual $\Lambda_{b}^{0}$ and 
$B^{0}$ reflections. A similar fit is performed for the $m\left(\psi(2 S) \Lambda^{0}\right)$ and $m\left(\psi(2 S) K_{S}^{0}\right)$ distributions. The projections of such fits for $\Lambda_{b}^{0} \rightarrow \psi(2 S) \Lambda^{0}$ and $B^{0} \rightarrow \psi(2 S) K_{S}^{0}$ are also shown in Fig. 1. Using the event yields extracted from these fits and corrected for the detector acceptance and reconstruction efficiency effects the following value of the branching ratio of the two $\Lambda_{b}^{0}$ decays was obtained: $\Gamma\left(\Lambda_{b}^{0} \rightarrow \psi(2 S) \Lambda^{0}\right) / \Gamma\left(\Lambda_{b}^{0} \rightarrow J / \psi \Lambda^{0}\right)=0.501 \pm 0.033$ (stat) \pm 0.016 (syst) $\pm 0.011(\mathscr{B})$. The measured ratio lies in the range $0.5-0.8$ found for the analogous $B$ meson decays. The only available theoretical prediction of this quantity $(0.8 \pm 0.1[3])$ exceeds the measured value.

\section{Study of the $B_{c}^{+} \rightarrow J / \psi D_{s}^{+}$and $B_{c}^{+} \rightarrow J / \psi D_{s}^{*+}$ decays}

The decays $B_{c}^{+} \rightarrow J / \psi D_{s}^{+}$and $B_{c}^{+} \rightarrow J / \psi D_{s}^{*+}$ were studied using $4.9 \mathrm{fb}^{-1}$ and $20.6 \mathrm{fb}^{-1}$ of pp-collision data at $\sqrt{s}=7 \mathrm{TeV}$ and $8 \mathrm{TeV}$, respectively [4]. The signal candidates were identified through $J / \psi \rightarrow \mu^{+} \mu^{-}$and $D_{s}^{(*)+} \rightarrow \phi \pi^{+}\left(\gamma / \pi^{0}\right)$ decays, $\phi$ meson decaying into a pair of charged kaons and the neutral pion or photon from the $D_{s}^{*+}$ decay not being reconstructed. The yields of $B_{c}^{+} \rightarrow J / \psi D_{s}^{+}$and $B_{c}^{+} \rightarrow J / \psi D_{s}^{*+}$ decays along with the transverse polarisation fraction in $B_{c}^{+} \rightarrow J / \psi D_{s}^{*+}$ decay were measured using a two-dimensional likelihood fit involving the $B_{c}^{+}$reconstructed invariant mass and the angle between the $\mu^{+}$and $D_{s}^{+}$candidate momenta in the muon pair rest frame. The transverse polarisation fraction is determined to be $\Gamma_{ \pm \pm}\left(B_{c}^{+} \rightarrow J / \psi D_{s}^{*+}\right) / \Gamma\left(B_{c}^{+} \rightarrow J / \psi D_{s}^{*+}\right)=0.38 \pm 0.23$ (stat) \pm 0.07 (syst) and the ratio of the branching fractions of the two modes is $\mathscr{B}\left(B_{c}^{+} \rightarrow J / \psi D_{s}^{*+}\right) / \mathscr{B}\left(B_{c}^{+} \rightarrow J / \psi D_{s}^{+}\right)=2.8_{-0.8}^{+1.2}$ (stat $) \pm$ 0.3 (syst). A sample of $B_{c}^{+} \rightarrow J / \psi \pi^{+}$decays was used to measure the ratios of branching fractions $\mathscr{B}\left(B_{c}^{+} \rightarrow J / \psi D_{s}^{+}\right) / \mathscr{B}\left(B_{c}^{+} \rightarrow J / \psi \pi^{+}\right)=3.8 \pm 1.1$ (stat) \pm 0.4 (syst) $\pm 0.2(\mathscr{B})$ and $\mathscr{B}\left(B_{c}^{+} \rightarrow J / \psi D_{s}^{*+}\right) / \mathscr{B}\left(B_{c}^{+} \rightarrow J / \psi \pi^{+}\right)=10.4 \pm 3.1$ (stat) \pm 1.5 (syst) $\pm 0.6(\mathscr{B})$. These results are shown in Fig. 2 compared with LHCb [5] and a number of theoretical predictions. As one can see, ATLAS result agrees with LHCb and is in general consistent with the available theoretical predictions.

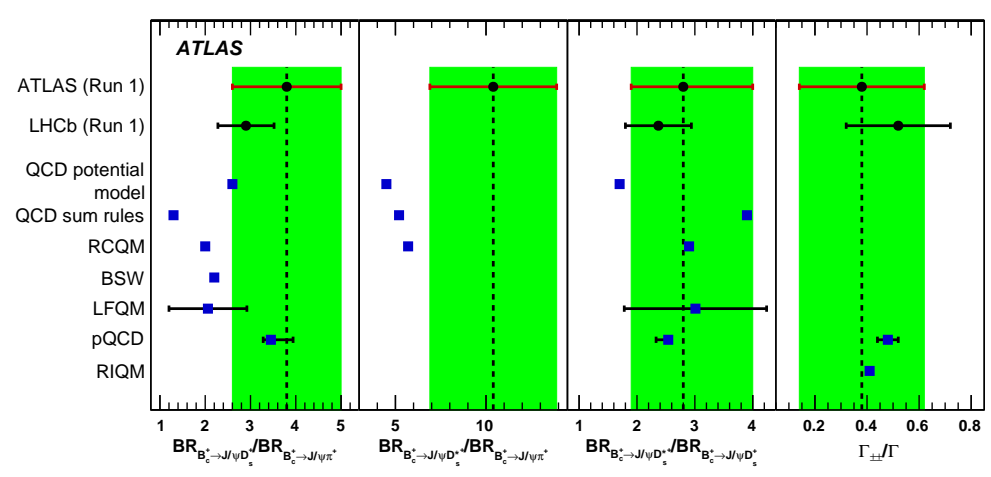

Figure 2: Comparison of the ATLAS measurement result for the relative branching fractions and polarisation in decays $B_{c}^{+} \rightarrow J / \psi D_{s}^{*+}$ and $B_{c}^{+} \rightarrow J / \psi \pi^{+}$with those of LHCb [5] and a number of theoretical predictions (for the full list of references see [4]).

\section{Determination of the ratio of $b$-quark fragmentation fractions $f_{s} / f_{d}$}

The ratio of $b$-quark fragmentation fractions was measured in ATLAS using $2.47 \mathrm{fb}^{-1}$ of $p p$ col- 
lisions at $\sqrt{s}=7 \mathrm{TeV}$ with the use of $B_{s}^{0} \rightarrow J / \psi\left(\mu^{+} \mu^{-}\right) \phi\left(K^{+} K^{-}\right)$and $B_{d}^{0} \rightarrow J / \psi\left(\mu^{+} \mu^{-}\right) K^{* 0}\left(K^{+} \pi^{-}\right)$ channels [6]. To investigate the $\eta$ and $p_{\mathrm{T}}$ dependencies, the measurement was performed in six $p_{\mathrm{T}}$ bins in the range $8 \mathrm{GeV}<p_{\mathrm{T}}<50 \mathrm{GeV}$ and in four $\eta$ bins in $|\eta|<2.5$. No significant $f_{s} / f_{d}$ dependence on $p_{\mathrm{T}}$ and $|\eta|$ can be seen at the present level of accuracy. For the case of $p_{\mathrm{T}}$ that can be illustrated with the Fig. 3(a), which shows the $p_{\mathrm{T}}$ dependence of $f_{s} / f_{d}$ for ATLAS and that of other experiments. Figure 3(b) shows the $f_{s} / f_{d}$ value measured by ATLAS compared with the other experiments. These results agree reasonably well.

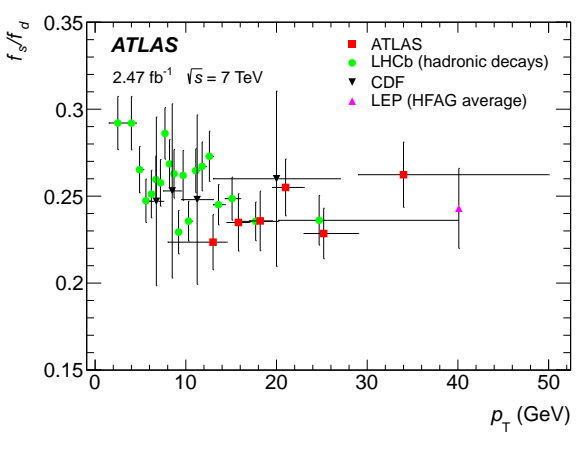

(a)

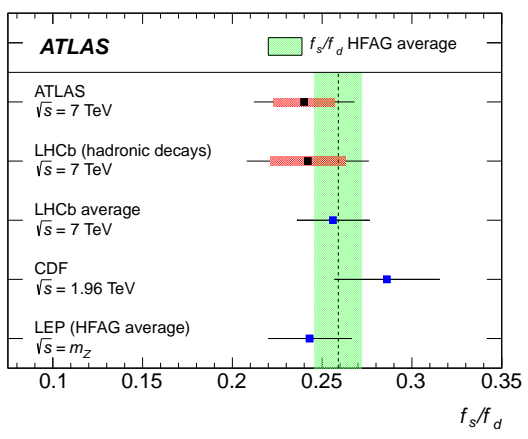

(b)

Figure 3: (a) Measurements of $f_{s} / f_{d}$ versus $B$ meson $p_{\mathrm{T}}$ for CDF, LHCb and ATLAS; (b) Measurements of $f_{s} / f_{d}$ from LEP, CDF, LHCb and ATLAS (for the full list of references see [6]).

\section{4. $B^{+}$mass reconstruction in $B^{+} \rightarrow J / \psi K^{+}$decay at $\sqrt{s}=13 \mathrm{TeV}$}

The performance of the ATLAS detector in reconstructing $B^{+}$events was tested using $3.2 \mathrm{fb}^{-1}$ of $p p$ collision data at $\sqrt{s}=13 \mathrm{TeV}$ [7]. Events with at least two oppositely charged muons were selected for this study. The two muon tracks were fitted into a common vertex to form a $J / \psi$ candidate. One more charged track, which was not identified as a muon, was then taken and fitted with the two muon tracks into a common vertex to form a $B^{+}$candidate. The invariant mass distribution of the selected $B^{+}$candidates was then fitted using an unbinned maximum likelihood fit with one signal and three background components. The three background components are: combinatorial, from the partially reconstructed decays, and a resonant component from the $B^{+} \rightarrow J / \psi \pi^{+}$decay. All the components but the $B^{+} \rightarrow J / \psi \pi^{+}$were described by analytical functions with free parameters, while the line shape of $B^{+} \rightarrow J / \psi \pi^{+}$was derived from MC and its yield relative to the signal was deduced from the measurement of branching ratio $\mathscr{B}\left(B^{+} \rightarrow J / \psi K^{+}\right) / \mathscr{B}\left(B^{+} \rightarrow J / \psi \pi^{+}\right)$by $\mathrm{LHCb}[8]$ and from the reconstruction efficiency ratio for these two channels obtained from MC. Figure 4(a) shows the invariant mass distribution for the selected $B^{+}$events along with the different fit components. It was decided to perform this fit independently in the intervals of rapidity of the $B^{+}$ candidates to account for the changes in the mass resolution. The fitted $B^{+}$mass values are shown in Fig. 4(b) as a function of $B^{+}$rapidity. One can see that the deviation of each measurement from the weighted mean value of the mass is less than $0.1 \%$, which indicates an excellent momentum calibration of the ATLAS Inner Detector.

An additional test of the fit was performed by imposing a cut on the transverse decay length $\left(L_{x y}\right)$ of the $B^{+}$candidates in order to change the background composition. The $B^{+}$mass values 


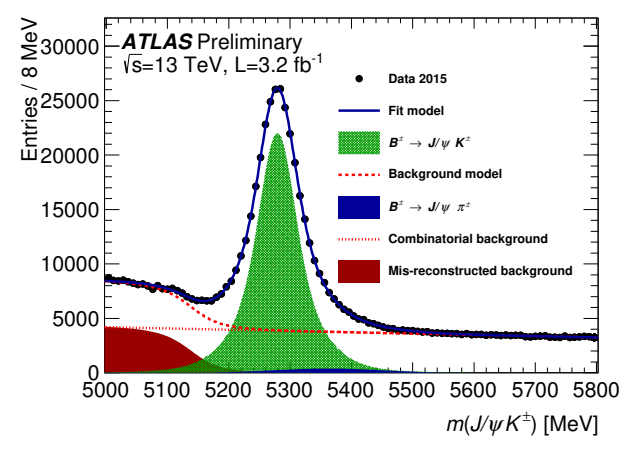

(a)

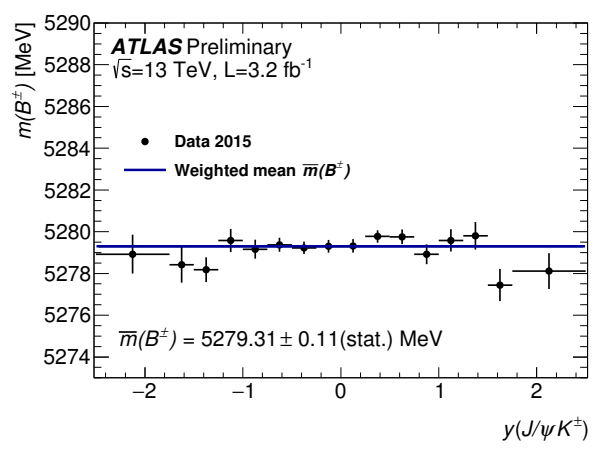

(b)

Figure 4: (a) $B^{+}$invariant mass distribution and the maximum likelihood fit components; (b) $B^{+}$mass values extracted from the fits as a function of rapidity [7].

measured by the two versions of the fit are shown in table 1 compared with a measurement by LHCb [9] and with the world average [10]. One can see that all the four numbers are in good agreement. The systematic uncertainty of the ATLAS measurement is partially evaluated to be $0.25 \mathrm{MeV}$, while that value does not yet include the uncertainty related to the momentum scale of the ATLAS Inner Detector.

\begin{tabular}{l|c|c}
\hline \hline Fit & $B^{+}$mass $[\mathrm{MeV}]$ & Fit error $[\mathrm{MeV}]$ \\
\hline Default fit & 5279.31 & 0.11 (stat.) \\
$L_{x y}>0.2 \mathrm{~mm}$ & 5279.34 & 0.09 (stat.) \\
\hline World Average fit & 5279.29 & 0.15 \\
LHCb & 5279.38 & 0.11 (stat.) \pm 0.33 (syst.) \\
\hline \hline
\end{tabular}

Table 1: $B^{+}$mass value measured in two ways [7] compared with the world average [10] and LHCb [9].

\section{Measurement of the differential non-prompt $J / \psi$ production fraction in $\sqrt{s}=13 \mathrm{TeV} p p$ collisions at the ATLAS experiment}

The non-prompt $J / \psi$ production fraction $\sigma\left(p p \rightarrow b+X \rightarrow J / \psi+X^{\prime}\right) / \sigma\left(p p \rightarrow J / \psi+X^{\prime}\right)$ was measured in $p p$ collisions at $\sqrt{s}=13$ with a data sample corresponding to an integrated luminosity of $6.4 \mathrm{pb}^{-1} \mathrm{TeV}$ [11]. The $J / \psi$ candidates were reconstructed in the $\mu^{+} \mu^{-}$channel and their invariant mass and pseudo-proper decay time ${ }^{1}$ distributions were simultaneously fitted using an unbinned maximum likelihood fit. The extracted non-prompt fraction values are shown in Fig. 5. One can see that the fraction grows with $p_{\mathrm{T}}$ of the $J / \psi$ candidates, while no rapidity dependence can be observed at the current level of precision.

\section{Conclusion}

This paper has presented several recent ATLAS results using both Run-1 and Run- 2 data. The results based on Run-1 data include observation and branching ratio measurement for $\Lambda_{b}^{0} \rightarrow \psi(2 S) \Lambda^{0}$

${ }^{1}$ Pseudo-proper decay time is defined as $\tau=L_{x y} \cdot \frac{m_{J / \psi}}{p_{\mathrm{T}}}$ 


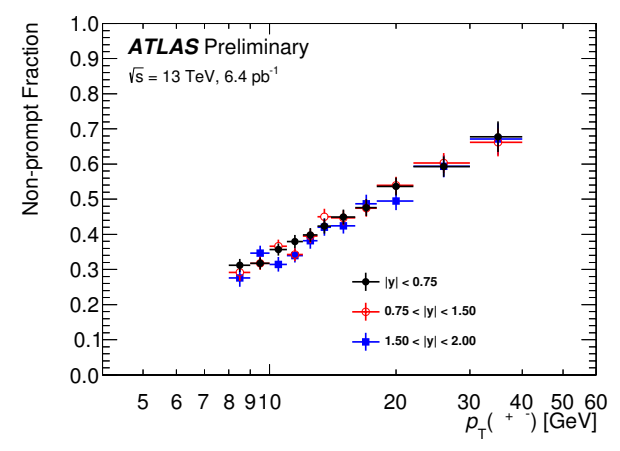

(a)

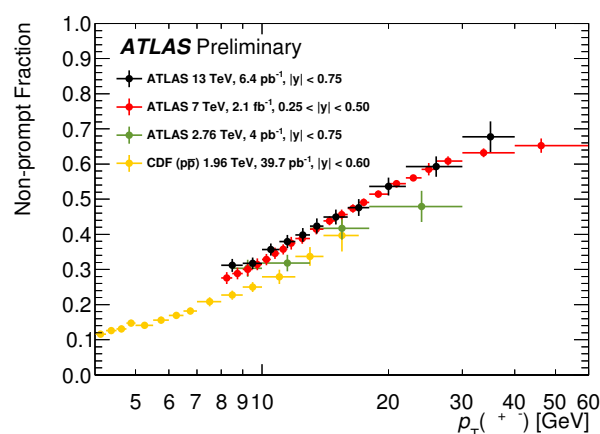

(b)

Figure 5: Measured non-prompt $J / \psi$ production fraction as a function of $J / \psi p_{\mathrm{T}}$ for three rapidity regions (a), and for the central rapidity region (b) compared with earlier measurments by ATLAS and CDF (for the complete list of references see [11]).

decay, the measurement of the branching fractions $\mathscr{B}\left(B_{c}^{+} \rightarrow J / \psi D_{s}^{(*)+}\right)$ and transverse polarisation fraction for the $B_{c}^{+} \rightarrow J / \psi D_{s}^{*+}$ decay, and the measurement of the $b$-quark fragmentation fractions ratio $f_{s} / f_{d}$. These results are in general consistent with other experiments, while the available prediction for $\Gamma\left(\Lambda_{b}^{0} \rightarrow \psi(2 S) \Lambda^{0}\right) / \Gamma\left(\Lambda_{b}^{0} \rightarrow J / \psi \Lambda^{0}\right)$ overestimates the measured value. Good performance of the ATLAS detector in Run-2 has been shown with two results using $\sqrt{s}=13 \mathrm{TeV}$ data: non-promt $J / \psi$ production fraction measurement and the measurement of $B^{+}$-meson mass, the latter demonstrating an excellent momentum calibration of the ATLAS Inner Detector.

\section{References}

[1] ATLAS Collaboration, JINST 3 (2008) S08003.

[2] ATLAS Collaboration, Phys. Lett. B751 (2015) 63-80, arXiv: 1507.08202 [hep-ex].

[3] T. Gutsche, M. A. Ivanov, J. G. Korner, V. E. Lyubovitskij, and P. Santorelli, Phys. Rev. D88 (2013) 114018, arXiv: 1309.7879 [hep-ph].

[4] ATLAS Collaboration, Eur. Phys. J. C76 (2016) 4, arXiv: 1507 .07099 [hep-ex].

[5] LHCb Collaboration, R. Aaij et al., Phys. Rev. D87 (2013) 112012, arXiv: 1304.4530 [hep-ex], [Addendum: Phys. Rev.D89,no.1,019901(2014)].

[6] ATLAS Collaboration, Phys. Rev. Lett. 115 (2015) 262001, arXiv: 1507.08925 [hep-ex].

[7] ATLAS Collaboration, ATLAS-CONF-2015-064 (2015), https://cds. cern.ch/record/2114830.

[8] LHCb Collaboration, R. Aaij et al., Phys. Rev. D85 (2012) 091105.

[9] LHCb Collaboration, R. Aaij et al., Phys. Lett. B708 (2012) 241-248.

[10] Particle Data Group Collaboration, K. Olive et al., Chinese Physics C 38 (2014) 090001.

[11] ATLAS Collaboration, ATLAS-CONF-2015-030 (2015), https://cds. cern.ch/record/2037967. 